Research Article

\title{
Some $L^{p}$ Inequalities for Polynomials Not Vanishing inside a Circle
}

\author{
Abdullah Mir and Bilal Dar \\ Department of Mathematics, University of Kashmir, Hazratbal Srinagar 190006, India \\ Correspondence should be addressed to Bilal Dar; darbilal85@ymail.com
}

Received 18 January 2014; Accepted 16 February 2014; Published 19 May 2014

Academic Editors: K. A. Lurie and M. A. McKibben

Copyright (C) 2014 A. Mir and B. Dar. This is an open access article distributed under the Creative Commons Attribution License, which permits unrestricted use, distribution, and reproduction in any medium, provided the original work is properly cited.

If $P(z)$ is a polynomial of degree $n$ having no zeros in $|z|<1$, then it is known that, for all $\alpha, \beta \in \mathscr{C}$ with $|\alpha| \leq 1,|\beta| \leq 1, R>r \geq 1$, and $p>0,\left\|P(R z)-\alpha P(r z)+\beta\left\{((R+1) /(r+1))^{n}-|\alpha|\right\} P(r z)\right\|_{p} \leq$ $\left(\left\|\left[R^{n}-\alpha r^{n}+\beta\left\{((R+1) /(r+1))^{n}-|\alpha|\right\}\right] z+\left[1-\alpha+\beta\left\{((R+1) /(r+1))^{n}-|\alpha|\right\}\right]\right\|_{p} /\|1+z\|_{p}\right)\|P(z)\|_{p}$. In this paper, we will prove a result which not only generalizes the above inequality but also generalize and refines the various results pertaining to the $L^{p}$ norm of $P(z) \forall p>0$. We will also prove a result which extends and refines a result of Boas Jr. and Rahman (1962). Also we will see that our results lead to some striking conclusions giving refinements and generalizations of other well-known results.

\section{Introduction}

For an $n$th degree polynomial $P(z)$, define

$$
\begin{aligned}
\|P\|_{p} & :=\left\{\frac{1}{2 \pi} \int_{0}^{2 \pi}\left|P\left(e^{i \theta}\right)\right|^{p}\right\}^{1 / p}, \quad p>0, \\
\|P\|_{\infty} & :=\operatorname{Max}_{|z|=1}|P(z)|, \quad m=\underset{|z|=1}{\operatorname{Min}}|P(z)| .
\end{aligned}
$$

If $P(z)$ is a polynomial of degree $n$, then

$$
\begin{gathered}
\left\|P^{\prime}\right\|_{\infty} \leq n\|P\|_{\infty}, \\
\|P(R z)\|_{\infty} \leq R^{n}\|P\|_{\infty}, \quad R>1 .
\end{gathered}
$$

Inequality (2) is an immediate consequence of a famous result due to Bernstein [1] on the derivative of a trigonometric polynomial, whereas inequality (3) is a simple deduction from the maximum modulus principle [2, page 346].

Restricting ourselves to a class of polynomials having no zero in $|z|<1$, the inequalities (2) and (3) can be, respectively, replaced by

$$
\begin{gathered}
\left\|P^{\prime}\right\|_{\infty} \leq \frac{n}{2}\|P\|_{\infty}, \\
\|P(R z)\|_{\infty} \leq \frac{R^{n}+1}{2}\|P\|_{\infty}, \quad R>1 .
\end{gathered}
$$

Inequality (4) was conjectured by Erdös and later verified by Lax [3], whereas Ankeny and Rivilin [4] used (4) to prove (5). Inequalities (4) and (5) were further improved in [5], where under the same hypothesis it was shown that

$$
\begin{gathered}
\left\|P^{\prime}\right\|_{\infty} \leq \frac{n}{2}\left(\|P\|_{\infty}-m\right), \\
\|P(R z)\|_{\infty} \leq \frac{R^{n}+1}{2}\|P\|_{\infty}-\frac{R^{n}-1}{2} m, \quad R>1 .
\end{gathered}
$$

Both inequalities (4) and (5) were generalized by Jain [6], who proved that if $P(z) \neq 0$ in $|z|<1$, then for every $\beta \in C$ with $|\beta| \leq 1$,

$$
\begin{array}{r}
\left|z P^{\prime}(z)+\frac{n \beta}{2} P(z)\right| \leq \frac{n}{2}\left(\left|1+\frac{\beta}{2}\right|+\left|\frac{\beta}{2}\right|\right)\|P\|_{\infty}, \\
\left|P(R z)+\beta\left(\frac{R+1}{2}\right)^{n} P(z)\right| \leq \frac{1}{2}\left[\left|R^{n}+\beta\left(\frac{R+1}{2}\right)^{n}\right|\right. \\
\left.+\left|1+\beta\left(\frac{R+1}{2}\right)^{n}\right|\right]\|P\|_{\infty},
\end{array}
$$

for $|z|=1$ and $R \geq 1$.

Further, Aziz and Rather [7] generalised inequality (8) by proving that if $P(z)$ is a polynomial of degree $n$ which does 
not vanish in $|z|<1$, then for all $\alpha, \beta \in \mathscr{C}$ with $|\alpha| \leq 1$, $|\beta| \leq 1$ and $R \geq 1$,

$$
\begin{gathered}
\left|P(R z)-\alpha P(z)+\beta\left\{\left(\frac{R+1}{2}\right)^{n}-|\alpha|\right\} P(z)\right| \\
\leq \frac{1}{2}\left[\left|R^{n}-\alpha+\beta\left\{\left(\frac{R+1}{2}\right)^{n}-|\alpha|\right\}\right||z|^{n}\right. \\
\left.+\left|1-\alpha+\beta\left\{\left(\frac{R+1}{2}\right)^{n}-|\alpha|\right\}\right|\right]\|P\|_{\infty} .
\end{gathered}
$$

The following result given in [8] provides a generalization of inequality (9) which interalia yields a compact generalization of inequality (8).

Theorem A. If $P(z)$ is a polynomial of degree $n$ which does not vanish in $|z|<1$, then for all $\alpha, \beta \in \mathscr{C}$ with $|\alpha| \leq 1,|\beta| \leq 1$, $R>r \geq 1$ and $|z| \geq 1$,

$$
\begin{array}{r}
\left|P(R z)-\alpha P(r z)+\beta\left\{\left(\frac{R+1}{r+1}\right)^{n}-|\alpha|\right\} P(r z)\right| \\
\leq \frac{1}{2}\left[\left|R^{n}-\alpha r^{n}+\beta\left\{\left(\frac{R+1}{r+1}\right)^{n}-|\alpha|\right\} r^{n}\right||z|^{n}\right. \\
\left.+\left|1-\alpha+\beta\left\{\left(\frac{R+1}{r+1}\right)^{n}-|\alpha|\right\}\right|\right]\|P\|_{\infty} .
\end{array}
$$

Further the following result given in [8] provides a refinement of Theorem A which among other results provides a compact generalization of inequalities (6) and (7) as well.

Theorem B. If $P(z)$ is a polynomial of degree $n$ which does not vanish in $|z|<1$, then for all $\alpha, \beta \in \mathscr{C}$ with $|\alpha| \leq 1,|\beta| \leq 1$, $R>r \geq 1$ and $|z|=1$,

$$
\begin{aligned}
& \mid P(R z)- \alpha P(r z)+\beta\left\{\left(\frac{R+1}{r+1}\right)^{n}-|\alpha|\right\} P(r z) \mid \\
& \leq \frac{1}{2}\left[\left\{\left|R^{n}-\alpha r^{n}+\beta\left\{\left(\frac{R+1}{r+1}\right)^{n}-|\alpha|\right\} r^{n}\right|\right.\right. \\
&\left.+\left|1-\alpha+\beta\left\{\left(\frac{R+1}{r+1}\right)^{n}-|\alpha|\right\}\right|\right\}\|P\|_{\infty} \\
&-\left\{\left|R^{n}-\alpha r^{n}+\beta\left\{\left(\frac{R+1}{r+1}\right)^{n}-|\alpha|\right\} r^{n}\right|\right. \\
&\left.\left.-\left|1-\alpha+\beta\left\{\left(\frac{R+1}{r+1}\right)^{n}-|\alpha|\right\}\right|\right\} m\right] .
\end{aligned}
$$

Recently, the dependence of $\| P(R z)-\alpha P(r z)+\beta$ $\left\{((R+1) /(r+1))^{n}-|\alpha|\right\} P(r z) \|_{p}$ on $\|P(z)\|_{p}$, was investigated in [9] for arbitrary complex numbers $\alpha, \beta$ with $|\alpha| \leq 1,|\beta| \leq 1, R>r \geq 1, p>0$ and the following compact generalization of Theorem A was proved.
Theorem C. If $P(z)$ is a polynomial of degree $n$ which does not vanish in $|z|<1$, then for all $\alpha, \beta \in \mathscr{C}$ with $|\alpha| \leq 1,|\beta| \leq 1$, $R>r \geq 1$ and $p>0$,

$$
\begin{gathered}
\left\|P(R z)-\alpha P(r z)+\beta\left\{\left(\frac{R+1}{r+1}\right)^{n}-|\alpha|\right\} P(r z)\right\|_{p} \\
\leq\left(\|\left[R^{n}-\alpha r^{n}+\beta\left\{\left(\frac{R+1}{r+1}\right)^{n}-|\alpha|\right\}\right] z\right. \\
+\left[1-\alpha+\beta\left\{\left(\frac{R+1}{r+1}\right)^{n}-|\alpha|\right\}\right] \|_{p} \\
\left.\times\left(\|1+z\|_{p}\right)^{-1}\right)\|P(z)\|_{p} .
\end{gathered}
$$

In this paper, we first prove the following more general result analogous to Theorem $\mathrm{C}$ which not only generalizes Theorem $\mathrm{B}$ to the $L^{p}$-norm of $P(z)$ for every $p>0$ but also leads to some striking conclusions giving refinements and generalizations of other well-known results.

Theorem 1. If $P(z)$ is a polynomial of degree $n$ which does not vanish in $|z|<1$, then for every $\alpha, \beta, \delta \in \mathscr{C}$ with $|\alpha| \leq 1$, $|\beta| \leq 1,|\delta| \leq 1, R>r \geq 1$, and $p>0$,

$$
\begin{aligned}
\| P(R z)-\alpha P(r z)+\beta\left\{\left(\frac{R+1}{r+1}\right)^{n}-|\alpha|\right\} P(r z) \\
+\frac{m \delta}{2}\left[\left|R^{n}-\alpha r^{n}+\beta\left\{\left(\frac{R+1}{r+1}\right)^{n}-|\alpha|\right\} r^{n}\right|\right. \\
\left.\quad-\left|1-\alpha+\beta\left\{\left(\frac{R+1}{r+1}\right)^{n}-|\alpha|\right\}\right|\right] \|_{p} \\
\leq\left(\|\left[R^{n}-\alpha r^{n}+\beta\left\{\left(\frac{R+1}{r+1}\right)^{n}-|\alpha|\right\}\right] z\right. \\
+\left[1-\alpha+\beta\left\{\left(\frac{R+1}{r+1}\right)^{n}-|\alpha|\right\}\right] \|_{p} \\
\left.\times\left(\|1+z\|_{p}\right)^{-1}\right)\|P(z)\|_{p} .
\end{aligned}
$$

The result is best possible and equality holds in (13) for the polynomial $P(z)=z^{n}+1$.

A variety of interesting results can be easily deduced from Theorem 1; here we mention few of them.

For $\delta=0$, Theorem 1 reduces to Theorem C. Also for $\delta=$ $\beta=0$, Theorem 1 reduces to a result of Aziz and Rather [10]. 
Further, on applying Minkowski's inequality on the right hand side of (13), we obtain, for $p \geq 1$,

$$
\begin{aligned}
& \| P(R z)-\alpha P(r z)+\beta\left\{\left(\frac{R+1}{r+1}\right)^{n}-|\alpha|\right\} P(r z) \\
& +\frac{m \delta}{2}\left[\left|R^{n}-\alpha r^{n}+\beta\left\{\left(\frac{R+1}{r+1}\right)^{n}-|\alpha|\right\} r^{n}\right|\right. \\
& \left.\quad-\left|1-\alpha+\beta\left\{\left(\frac{R+1}{r+1}\right)^{n}-|\alpha|\right\}\right|\right] \|_{p} \\
& \leq\left|R^{n}-\alpha r^{n}+\beta r^{n}\left\{\left(\frac{R+1}{r+1}\right)^{n}-|\alpha|\right\}\right| \\
& +\left|1-\alpha+\beta\left\{\left(\frac{R+1}{r+1}\right)^{n}-|\alpha|\right\}\right| \\
& \quad \times\left(\|1+z\|_{p}\right)^{-1}\|P(z)\|_{p} .
\end{aligned}
$$

Now by taking $\delta=0$ and $r=1$ in the above inequality and then letting $p \rightarrow \infty$, we get inequality (9).

Also by taking $\delta=0$ in inequality (14) and making $p \rightarrow$ $\infty$, we obtain Theorem $A$.

The following corollary which is a compact generalization of (7) follows from Theorem 1 by taking $\beta=0$.

Corollary 2. If $P(z)$ is a polynomial of degree $n$ which does not vanish in $|z|<1$, then for every $\alpha, \delta \in \mathscr{C}$ with $|\alpha| \leq 1,|\delta| \leq 1$, $R>r \geq 1$, and $p>0$,

$$
\begin{gathered}
\left\|P(R z)-\alpha P(r z)+\frac{m \delta}{2}\left(\left|R^{n}-\alpha r^{n}\right|-|1-\alpha|\right)\right\|_{p} \\
\leq \frac{\left\|\left(R^{n}-\alpha r^{n}\right) z-(1-\alpha)\right\|_{p}}{\|1+z\|_{p}}\|P(z)\|_{p} .
\end{gathered}
$$

The result is best possible and equality holds in (15) for the polynomial $P(z)=z^{n}+1$.

Remark 3. For $\delta=0$, Corollary 2 reduces to a result of Aziz and Rather [11]. For $\delta=0$ and $r=\alpha=1$, Corollary 2 reduces to a result of Aziz and Rather [12]. Again for $\alpha=0$ and $r=1$, we get a result recently proved by Rather [13, Theorem 1.1].

Finally, as an application of Theorem 1, we prove the following generalization and refinement of a result of Boas Jr. and Rahman [14] for $p \geq 1$.

Theorem 4. If $P(z)$ is a polynomial of degree $n$ which does not vanish in $|z|<1$, then for every $\delta, \alpha \in \mathscr{C}$ with $|\delta| \leq 1,|\alpha| \leq 1$ and $R>r \geq 1, p \geq 1$,

$$
\begin{aligned}
& \left\|p(R z)+\delta\left(\frac{\left|R^{n}-\alpha r^{n}\right|-|1-\alpha|}{2}\right) m\right\|_{p} \\
& \leq\left\{|\alpha| r^{n}+\frac{\left\|\left(R^{n}-\alpha r^{n}\right) z+(1-\alpha)\right\|_{p}}{\|1+z\|_{p}}\right\}\|p(z)\|_{p} .
\end{aligned}
$$

For $\delta=\alpha=0$, Theorem 4 reduces to a result of Boas Jr. and Rahman [14] for $p \geq 1$. Also for $\alpha=r=1$, Theorem 4 reduces to the following corollary which is a compact generalization of inequality (7) due to Aziz and Dawood [5, Theorem 2] to $L^{p}$ norm.

Corollary 5. If $P(z)$ is a polynomial of degreen which does not vanish in $|z|<1$, then for every $\delta \in \mathscr{C}$ with $|\delta| \leq 1, R>1$ and $p \geq 1$,

$$
\begin{gathered}
\left\|p(R z)+\delta\left(\frac{R^{n}-1}{2}\right) m\right\|_{p} \\
\quad \leq\left\{1+\frac{\left(R^{n}-1\right)}{\|1+z\|_{p}}\right\}\|p(z)\|_{p} .
\end{gathered}
$$

\section{Lemmas}

For the proof of these theorems we need the following lemmas.

Lemma 6. If $f(z)$ is a polynomial of degree $n$ having all its zeros in $|z| \leq 1$, then for all $\alpha, \beta \in \mathscr{C}$ with $|\alpha| \leq 1,|\beta| \leq 1$ and $R>r \geq 1$,

$$
\begin{aligned}
& \underset{|z|=1}{\operatorname{Min}}\left|f(R z)-\alpha f(r z)+\beta\left\{\left(\frac{R+1}{r+1}\right)^{n}-|\alpha|\right\} f(r z)\right| \\
& \geq\left|R^{n}-\alpha r^{n}+\beta\left\{\left(\frac{R+1}{r+1}\right)^{n}-|\alpha|\right\} r^{n}\right| \operatorname{Min}_{|z|=1}|f(z)| .
\end{aligned}
$$

Lemma 7. If $f(z)$ is a polynomial of degree $n$ which does not vanish in $|z|<1$, then for all $\alpha, \beta \in \mathscr{C}$ with $|\alpha| \leq 1,|\beta| \leq 1$, and $R>r \geq 1$,

$$
\begin{aligned}
& \left|f(R z)-\alpha f(r z)+\beta\left\{\left(\frac{R+1}{r+1}\right)^{n}-|\alpha|\right\} f(r z)\right| \\
& \quad \leq\left|g(R z)-\alpha g(r z)+\beta\left\{\left(\frac{R+1}{r+1}\right)^{n}-|\alpha|\right\} g(r z)\right|,
\end{aligned}
$$

where $g(z)=z^{n} \overline{f(1 / \bar{z})}$.

The above two lemmas are proved in [8].

Lemma 8. If $f(z)$ is a polynomial of degree $n$ which does not vanish in $|z|<1$, then for all $\alpha, \beta \in \mathscr{C}$ with $|\alpha| \leq 1,|\beta| \leq 1$ and $R>r \geq 1$,

$$
\begin{aligned}
& \left|f(R z)-\alpha f(r z)+\beta\left\{\left(\frac{R+1}{r+1}\right)^{n}-|\alpha|\right\} f(r z)\right| \\
& \leq\left|g(R z)-\alpha g(r z)+\beta\left\{\left(\frac{R+1}{r+1}\right)^{n}-|\alpha|\right\} g(r z)\right| \\
& -\left\{\left|R^{n}-\alpha r^{n}+\beta\left\{\left(\frac{R+1}{r+1}\right)^{n}-|\alpha|\right\} r^{n}\right|\right. \\
& \left.-\left|1-\alpha+\beta\left\{\left(\frac{R+1}{r+1}\right)^{n}-|\alpha|\right\}\right|\right\} \underset{|z|=1}{\operatorname{Min}|f(z)|,}
\end{aligned}
$$

where $g(z)=z^{n} \overline{f(1 / \bar{z})}$. 
Proof of Lemma 8. If $f(z)$ has a zero on $|z|=1$, then $m=$ $\operatorname{Min}_{|z|=1}|f(z)|=0$ and the result follows from Lemma 7 . Therefore, we assume that $f(z)$ has all its zeros in $|z|>1$, so that $m>0$. Now for any $\delta \in \mathscr{C}$ with $|\delta|<1$, we have $|\delta m|<$ $m \leq|f(z)|$, for $|z|=1$. By Rouche's theorem, the polynomial $H(z)=f(z)-\delta m$ has no zero in $|z|<1$. If $g(z)=z^{n} \overline{f(1 / \bar{z})}$, then the polynomial $G(z)=z^{n} \overline{H(1 / \bar{z})}=g(z)-\bar{\delta} m z^{n}$ has all its zeros in $|z| \leq 1$ and also $|H(z)|=|G(z)|$ on $|z|=1$. Therefore by Lemma 7, for all $\alpha, \beta \in \mathscr{C}$ with $|\alpha| \leq 1,|\beta| \leq 1$ and $R>r \geq 1$, we have

$$
\begin{array}{r}
\left|H(R z)-\alpha H(r z)+\beta\left\{\left(\frac{R+1}{r+1}\right)^{n}-|\alpha|\right\} H(r z)\right| \\
\leq\left|G(R z)-\alpha G(r z)+\beta\left\{\left(\frac{R+1}{r+1}\right)^{n}-|\alpha|\right\} G(r z)\right|, \\
\text { for }|z|=1 .
\end{array}
$$

Equivalently,

$$
\begin{aligned}
& \mid f(R z)-\alpha f(r z)+\beta\left\{\left(\frac{R+1}{r+1}\right)^{n}-|\alpha|\right\} f(r z) \\
& -m \delta\left\{1-\alpha+\beta\left(\left(\frac{R+1}{r+1}\right)^{n}-|\alpha|\right)\right\} \mid \\
& \leq \mid g(R z)-\alpha g(r z)+\beta\left\{\left(\frac{R+1}{r+1}\right)^{n}-|\alpha|\right\} g(r z) \\
& \quad-m \bar{\delta}\left\{R^{n}-\alpha r^{n}+\beta\left(\left(\frac{R+1}{r+1}\right)^{n}-|\alpha|\right) r^{n}\right\} z^{n} \mid .
\end{aligned}
$$

Now choosing the argument of $\delta$ on the right hand side of (22) such that

$$
\begin{aligned}
& \mid g(R z)-\alpha g(r z)+\beta\left\{\left(\frac{R+1}{r+1}\right)^{n}-|\alpha|\right\} g(r z) \\
& -m \bar{\delta}\left\{R^{n}-\alpha r^{n}+\beta\left(\left(\frac{R+1}{r+1}\right)^{n}-|\alpha|\right) r^{n}\right\} z^{n} \mid \\
& =\left|g(R z)-\alpha g(r z)+\beta\left\{\left(\frac{R+1}{r+1}\right)^{n}-|\alpha|\right\} g(r z)\right| \\
& \quad-m|\delta|\left|R^{n}-\alpha r^{n}+\beta\left(\left(\frac{R+1}{r+1}\right)^{n}-|\alpha|\right) r^{n}\right|
\end{aligned}
$$

which is possible by Lemma 6 and the fact that $\operatorname{Min}_{|z|=1}|f(z)|=\operatorname{Min}_{|z|=1}|g(z)|$, we get, for $|z|=1$,

$$
\begin{aligned}
& \left|f(R z)-\alpha f(r z)+\beta\left\{\left(\frac{R+1}{r+1}\right)^{n}-|\alpha|\right\} f(r z)\right| \\
& \quad-m|\delta|\left|1-\alpha+\beta\left(\left(\frac{R+1}{r+1}\right)^{n}-|\alpha|\right)\right| \\
& \leq\left|g(R z)-\alpha g(r z)+\beta\left\{\left(\frac{R+1}{r+1}\right)^{n}-|\alpha|\right\} g(r z)\right| \\
& \quad-m|\delta|\left|R^{n}-\alpha r^{n}+\beta\left(\left(\frac{R+1}{r+1}\right)^{n}-|\alpha|\right) r^{n}\right| .
\end{aligned}
$$

Now, if in (24) we make $|\delta| \rightarrow 1$, we get

$$
\begin{gathered}
\left|f(R z)-\alpha f(r z)+\beta\left\{\left(\frac{R+1}{r+1}\right)^{n}-|\alpha|\right\} f(r z)\right| \\
\leq\left|g(R z)-\alpha g(r z)+\beta\left\{\left(\frac{R+1}{r+1}\right)^{n}-|\alpha|\right\} g(r z)\right| \\
-\left\{\left|R^{n}-\alpha r^{n}+\beta\left\{\left(\frac{R+1}{r+1}\right)^{n}-|\alpha|\right\} r^{n}\right|\right. \\
\left.-\left|1-\alpha+\beta\left\{\left(\frac{R+1}{r+1}\right)^{n}-|\alpha|\right\}\right|\right\} m,
\end{gathered}
$$

which is inequality (20) and that proves Lemma 8 completely.

Lemma 9. If $P(z)$ is a polynomial of degree $n$ having no zeros in $|z|<1$, then for every $\alpha, \beta \in \mathscr{C}$ with $|\alpha| \leq 1,|\beta| \leq 1$ and $R>r \geq 1, p>0$ and $\gamma$ real,

$$
\begin{aligned}
& \int_{0}^{2 \pi} \mid\left[P\left(R e^{i \theta}\right)-\alpha P\left(r e^{i \theta}\right)\right. \\
& \left.+\beta\left\{\left(\frac{R+1}{r+1}\right)^{n}-|\alpha|\right\} P\left(r e^{i \theta}\right)\right] \\
& +\quad e^{i \gamma}\left[R^{n} P\left(\frac{e^{i \theta}}{R}\right)-\bar{\alpha} r^{n} P\left(\frac{e^{i \theta}}{r}\right)\right. \\
& \left.\quad+\bar{\beta} r^{n}\left\{\left(\frac{R+1}{r+1}\right)^{n}|\alpha|\right\} P\left(\frac{e^{i \theta}}{r}\right)\right]\left.\right|^{p} d \theta \\
& +\quad R^{n}-\alpha r^{n}+\beta r^{n}\left\{\left(\frac{R+1}{r+1}\right)^{n}-|\alpha|\right\} \\
& +\left.e^{i \gamma}\left[1-\bar{\alpha}+\bar{\beta}\left\{\left(\frac{R+1}{r+1}\right)^{n}-|\alpha|\right\}\right]\right|^{p} \int_{0}^{2 \pi}\left|P\left(e^{i \theta}\right)\right|^{p} d \theta .
\end{aligned}
$$

The above lemma is proved in [9].

Lemma 10. If $A, B$, and $C$ are nonnegative real numbers such that $B+C \leq A$, then for every real number $\alpha$,

$$
\left|(A-C) e^{i \alpha}+(B+C)\right| \leq\left|A e^{i \alpha}+B\right| .
$$

The above lemma is due to Aziz and Rather [15].

Lemma 11. If $P(z)$ is a polynomial of degree $n$, then for every $R \geq 1$ and $p>0$,

$$
\left\{\int_{0}^{2 \pi}\left|P\left(R e^{i \theta}\right)\right|^{p}\right\}^{1 / p} \leq R^{n}\left\{\int_{0}^{2 \pi}\left|P\left(e^{i \theta}\right)\right|^{p}\right\}^{1 / p} .
$$

The above lemma is a simple consequence of a result of Hardy [16]. 
ISRN Mathematical Analysis

5

3. Proof of Theorems

Proof of Theorem 1. Since $P(z) \neq 0$ in $|z|<1$, therefore, by Lemma 8 , for each $\theta, 0 \leq \theta<2 \pi$ and for all $\alpha, \beta \in \mathscr{C}$ with $|\alpha| \leq 1,|\beta| \leq 1$ and $R>r \geq 1$, we have

$$
\begin{gathered}
\left|P\left(R e^{i \theta}\right)-\alpha P\left(r e^{i \theta}\right)+\beta\left\{\left(\frac{R+1}{r+1}\right)^{n}-|\alpha|\right\} P\left(r e^{i \theta}\right)\right| \\
\leq\left|Q\left(R e^{i \theta}\right)-\alpha Q\left(r e^{i \theta}\right)+\beta\left\{\left(\frac{R+1}{r+1}\right)^{n}-|\alpha|\right\} Q\left(r e^{i \theta}\right)\right| \\
-m\left\{\left|R^{n}-\alpha r^{n}+\beta\left\{\left(\frac{R+1}{r+1}\right)^{n}-|\alpha|\right\} r^{n}\right|\right. \\
\left.-\left|1-\alpha+\beta\left\{\left(\frac{R+1}{r+1}\right)^{n}-|\alpha|\right\}\right|\right\},
\end{gathered}
$$

where $m=\operatorname{Min}_{|z|=k}|P(z)|$ and $Q(z)=z^{n} \overline{P(1 / \bar{z})}$.

This implies that

$$
\begin{aligned}
& \left|P\left(R e^{i \theta}\right)-\alpha P\left(r e^{i \theta}\right)+\beta\left\{\left(\frac{R+1}{r+1}\right)^{n}-|\alpha|\right\} P\left(r e^{i \theta}\right)\right| \\
& \leq \mid R^{n} P\left(\frac{e^{i \theta}}{R}\right)-\bar{\alpha} r^{n} P\left(\frac{e^{i \theta}}{r}\right) \\
& +\bar{\beta}\left\{\left(\frac{R+1}{r+1}\right)^{n}-|\alpha|\right\} r^{n} P\left(\frac{e^{i \theta}}{r}\right) \mid \\
& -m\left\{\left|R^{n}-\alpha r^{n}+\beta\left\{\left(\frac{R+1}{r+1}\right)^{n}-|\alpha|\right\} r^{n}\right|\right. \\
& \left.-\left|1-\alpha+\beta\left\{\left(\frac{R+1}{r+1}\right)^{n}-|\alpha|\right\}\right|\right\},
\end{aligned}
$$

or

$$
\begin{gathered}
\left|P\left(R e^{i \theta}\right)-\alpha P\left(r e^{i \theta}\right)+\beta\left\{\left(\frac{R+1}{r+1}\right)^{n}-|\alpha|\right\} P\left(r e^{i \theta}\right)\right| \\
+\frac{m}{2}\left[\left|R^{n}-\alpha r^{n}+\beta\left\{\left(\frac{R+1}{r+1}\right)^{n}-|\alpha|\right\} r^{n}\right|\right. \\
\left.-\left|1-\alpha+\beta\left\{\left(\frac{R+1}{r+1}\right)^{n}-|\alpha|\right\}\right|\right] \\
\leq \mid R^{n} P\left(\frac{e^{i \theta}}{R}\right)-\bar{\alpha} r^{n} P\left(\frac{e^{i \theta}}{r}\right) \\
+\bar{\beta}\left\{\left(\frac{R+1}{r+1}\right)^{n}-|\alpha|\right\} r^{n} P\left(\frac{e^{i \theta}}{r}\right) \mid \\
-\frac{m}{2}\left[\left|R^{n}-\alpha r^{n}+\beta\left\{\left(\frac{R+1}{r+1}\right)^{n}-|\alpha|\right\} r^{n}\right|\right. \\
\left.-\left|1-\alpha+\beta\left\{\left(\frac{R+1}{r+1}\right)^{n}-|\alpha|\right\}\right|\right] .
\end{gathered}
$$

Taking

$$
\begin{gathered}
A=\mid R^{n} P\left(\frac{e^{i \theta}}{R}\right)-\bar{\alpha} r^{n} P\left(\frac{e^{i \theta}}{r}\right) \\
+\bar{\beta}\left\{\left(\frac{R+1}{r+1}\right)^{n}-|\alpha|\right\} r^{n} P\left(\frac{e^{i \theta}}{r}\right) \mid, \\
B=\mid P\left(R e^{i \theta}\right)-\alpha P\left(r e^{i \theta}\right) \\
+\beta\left\{\left(\frac{R+1}{r+1}\right)^{n}-|\alpha|\right\} P\left(r e^{i \theta}\right) \mid, \\
C=\frac{m}{2}\left[\left|R^{n}-\alpha r^{n}+\beta\left\{\left(\frac{R+1}{r+1}\right)^{n}-|\alpha|\right\} r^{n}\right|\right. \\
\left.-\left|1-\alpha+\beta\left\{\left(\frac{R+1}{r+1}\right)^{n}-|\alpha|\right\}\right|\right]
\end{gathered}
$$

in Lemma 10 and noting by (31) that $B+C \leq A-C \leq A$, we get, for every real $\gamma$,

$$
\begin{aligned}
& \mid\left\{\mid R^{n} P\left(\frac{e^{i \theta}}{R}\right)-\bar{\alpha} r^{n} P\left(\frac{e^{i \theta}}{r}\right)\right. \\
& +\bar{\beta}\left\{\left(\frac{R+1}{r+1}\right)^{n}-|\alpha|\right\} r^{n} P\left(\frac{e^{i \theta}}{r}\right) \mid \\
& -\frac{m}{2}\left[\left|R^{n}-\alpha r^{n}+\beta\left\{\left(\frac{R+1}{r+1}\right)^{n}-|\alpha|\right\} r^{n}\right|\right. \\
& +\left\{\mid P\left(R e^{i \theta}\right)-\alpha P\left(r e^{i \theta}\right)\right. \\
& +\beta\left\{\left(\frac{R+1}{r+1}\right)^{n}-|\alpha|\right\} P\left(r e^{i \theta}\right) \mid \\
& +\frac{m}{2}\left[\left|R^{n}-\alpha r^{n}+\beta\left\{\left(\frac{R+1}{r+1}\right)^{n}-|\alpha|\right\} r^{n}\right|\right. \\
& \left.\left.+\quad \bar{\beta}\left\{\left(\frac{R+1}{r+1}\right)^{n}-|\alpha|\right\} r^{n} P\left(\frac{e^{i \theta}}{r}\right)|\alpha|\right\} \mid\right] e^{i \gamma} \\
& \leq|| R^{n} P\left(\frac{e^{i \theta}}{R}\right)-\bar{\alpha} r^{n} P\left(\frac{e^{i \theta}}{r}\right) \\
& \left.\left.+\left|1-\alpha+\beta\left\{\left(\frac{R+1}{r+1}\right)^{n}-|\alpha|\right\}\right|\right]\right\} \mid
\end{aligned}
$$


This implies, for each $p>0$, that

$$
\begin{aligned}
& \int_{0}^{2 \pi}\left|F(\theta)+e^{i \gamma} G(\theta)\right|^{p} d \theta \\
& \leq \int_{0}^{2 \pi}|| R^{n} P\left(\frac{e^{i \theta}}{R}\right)-\bar{\alpha} r^{n} P\left(\frac{e^{i \theta}}{r}\right) \\
&+ \bar{\beta}\left\{\left(\frac{R+1}{r+1}\right)^{n}-|\alpha|\right\} r^{n} P\left(\frac{e^{i \theta}}{r}\right) \mid e^{i \gamma} \\
&+ \mid P\left(R e^{i \theta}\right)-\alpha P\left(r e^{i \theta}\right) \\
&+ \beta\left\{\left(\frac{R+1}{r+1}\right)^{n}-|\alpha|\right\} P\left(r e^{i \theta}\right) \|^{p} d \theta
\end{aligned}
$$

where

$$
\begin{gathered}
F(\theta)=\left|P\left(R e^{i \theta}\right)-\alpha P\left(r e^{i \theta}\right)+\beta\left\{\left(\frac{R+1}{r+1}\right)^{n}-|\alpha|\right\} P\left(r e^{i \theta}\right)\right| \\
+\frac{m}{2}\left[\left|R^{n}-\alpha r^{n}+\beta\left\{\left(\frac{R+1}{r+1}\right)^{n}-|\alpha|\right\} r^{n}\right|\right. \\
\left.\quad-\left|1-\alpha+\beta\left\{\left(\frac{R+1}{r+1}\right)^{n}-|\alpha|\right\}\right|\right], \\
G(\theta)=\mid R^{n} P\left(\frac{e^{i \theta}}{R}\right)-\bar{\alpha} r^{n} P\left(\frac{e^{i \theta}}{r}\right) \\
+\bar{\beta}\left\{\left(\frac{R+1}{r+1}\right)^{n}-|\alpha|\right\} r^{n} P\left(\frac{e^{i \theta}}{r}\right) \mid \\
-\frac{m}{2}\left[\left|R^{n}-\alpha r^{n}+\beta\left\{\left(\frac{R+1}{r+1}\right)^{n}-|\alpha|\right\} r^{n}\right|\right. \\
\left.\quad-\left|1-\alpha+\beta\left\{\left(\frac{R+1}{r+1}\right)^{n}-|\alpha|\right\}\right|\right] .
\end{gathered}
$$

Integrating both sides of (34) with respect to $\gamma$ from 0 to $2 \pi$, we get with the help of Lemma 9, for each $p>0, R>r \geq$ $1,|\alpha| \leq 1,|\beta| \leq 1$ and $\gamma$ real,

$$
\begin{aligned}
& \int_{0}^{2 \pi} \int_{0}^{2 \pi} \mid F(\theta)+\left.e^{i \gamma} G(\theta)\right|^{p} d \theta d \gamma \\
& \leq \int_{0}^{2 \pi}\left\{\int_{0}^{2 \pi}|| R^{n} P\left(\frac{e^{i \theta}}{R}\right)-\bar{\alpha} r^{n} P\left(\frac{e^{i \theta}}{r}\right)\right. \\
&+\bar{\beta}\left\{\left(\frac{R+1}{r+1}\right)^{n}-|\alpha|\right\} r^{n} P\left(\frac{e^{i \theta}}{r}\right) \mid e^{i \gamma} \\
&+\mid P\left(R e^{i \theta}\right)-\alpha P\left(r e^{i \theta}\right) \\
&\left.+\beta\left\{\left(\frac{R+1}{r+1}\right)^{n}-|\alpha|\right\} P\left(r e^{i \theta}\right) \mid \|^{p} d \theta\right\} d \gamma
\end{aligned}
$$

$$
\begin{aligned}
& =\int_{o}^{2 \pi}\left\{\int_{0}^{2 \pi} \mid\left(R^{n} P\left(\frac{e^{i \theta}}{R}\right)-\bar{\alpha} r^{n} P\left(\frac{e^{i \theta}}{r}\right)\right.\right. \\
& \left.+\bar{\beta}\left\{\left(\frac{R+1}{r+1}\right)^{n}-|\alpha|\right\} r^{n} P\left(\frac{e^{i \theta}}{r}\right)\right) e^{i \gamma} \\
& +\left(P\left(R e^{i \theta}\right)-\alpha P\left(r e^{i \theta}\right)\right. \\
& \left.\left.+\beta\left\{\left(\frac{R+1}{r+1}\right)^{n}-|\alpha|\right\} P\left(r e^{i \theta}\right)\right)\left.\right|^{p} d \theta\right\} d \gamma \\
& \leq \int_{0}^{2 \pi} \mid R^{n}-\alpha r^{n}+\beta r^{n}\left\{\left(\frac{R+1}{r+1}\right)^{n}-|\alpha|\right\} \\
& +\left.e^{i \gamma}\left[1-\bar{\alpha}+\bar{\beta}\left\{\left(\frac{R+1}{r+1}\right)^{n}-|\alpha|\right\}\right]\right|^{p} d \gamma \\
& \times \int_{0}^{2 \pi}\left|P\left(e^{i \theta}\right)\right|^{p} d \theta \text {. }
\end{aligned}
$$

Now for every real $\gamma$ and $t \geq 1$, we have

$$
\left|t+e^{i \gamma}\right| \geq\left|1+e^{i \gamma}\right|
$$

which implies, for $p>0$,

$$
\int_{0}^{2 \pi}\left|t+e^{i \gamma}\right|^{p} d \gamma \geq \int_{0}^{2 \pi}\left|1+e^{i \gamma}\right|^{p} d \gamma .
$$

If $F(\theta) \neq 0$, we take $t=|G(\theta) / F(\theta)|$; then from (31), we have $t \geq 1$; hence

$$
\begin{aligned}
\int_{0}^{2 \pi} & \left|F(\theta)+e^{i \gamma} G(\theta)\right|^{p} d \gamma \\
= & |F(\theta)|^{p} \int_{0}^{2 \pi}\left|1+\frac{G(\theta)}{F(\theta)} e^{i \gamma}\right|^{p} d \gamma \\
= & |F(\theta)|^{p} \int_{0}^{2 \pi}\left|\frac{G(\theta)}{F(\theta)}+e^{i \gamma}\right|^{p} d \gamma \\
= & |F(\theta)|^{p} \int_{0}^{2 \pi}|| \frac{G(\theta)}{F(\theta)}\left|+e^{i \gamma}\right|^{p} d \gamma \\
\geq & |F(\theta)|^{p} \int_{0}^{2 \pi}\left|1+e^{i \gamma}\right|^{p} d \gamma \\
= & \left\{\mid P\left(R e^{i \theta}\right)-\alpha P\left(r e^{i \theta}\right)\right. \\
& +\beta\left\{\int_{0}^{2 \pi}\left|1+e^{i \gamma}\right|^{p} d \gamma .\right. \\
& +\frac{m}{2}\left[\left|R^{n}-\alpha r^{n}+\beta\left\{\left(\frac{R+1}{r+1}\right)^{n}-|\alpha|\right\} r^{n}\right|\right. \\
& \left.\left.-\left|1-\alpha+\beta\left\{\left(\frac{R+1}{r+1}\right)^{n}-|\alpha|\right\}\right|\right]\right\}^{p} \\
& +|\alpha|\} P\left(r e^{i \theta}\right) \mid
\end{aligned}
$$


For $F(\theta)=0$, this inequality is trivially true. Using this in (36), we conclude that for all $\alpha, \beta \in \mathscr{C}$ with $|\alpha| \leq 1,|\beta| \leq 1$, $R>r \geq 1,0 \leq \theta<2 \pi$, and $p>0$,

$$
\begin{aligned}
& \int_{0}^{2 \pi}\left|1+e^{i \gamma}\right|^{p} d \gamma \\
& \times \int_{0}^{2 \pi}\left\{\mid P\left(R e^{i \theta}\right)-\alpha P\left(r e^{i \theta}\right)\right. \\
& +\beta\left\{\left(\frac{R+1}{r+1}\right)^{n}-|\alpha|\right\} P\left(r e^{i \theta}\right) \mid \\
& +\frac{m}{2}\left[\left|R^{n}-\alpha r^{n}+\beta\left\{\left(\frac{R+1}{r+1}\right)^{n}-|\alpha|\right\} r^{n}\right|\right. \\
& \leq \int_{0}^{2 \pi} \mid R^{n}-\alpha r^{n}+\beta r^{n}\left\{\left(\frac{R+1}{r+1}\right)^{n}-|\alpha|\right\} \\
& +e^{i \gamma}\left[1-\bar{\alpha}+\bar{\beta}\left\{\left(\frac{R+1}{r+1}\right)^{n}-|\alpha|\right\}||^{p} d \gamma\right. \\
& \times \int_{0}^{2 \pi} P\left|\left(e^{i \theta}\right)\right|^{p} d \theta .
\end{aligned}
$$

Now using the fact that for every complex number $\delta$ with $|\delta| \leq 1$,

$$
\begin{aligned}
& \mid P\left(R e^{i \theta}\right)-\alpha P\left(r e^{i \theta}\right)+\beta\left\{\left(\frac{R+1}{r+1}\right)^{n}-|\alpha|\right\} P\left(r e^{i \theta}\right) \\
& +\frac{m \delta}{2}\left(\left|R^{n}-\alpha r^{n}+\beta\left\{\left(\frac{R+1}{r+1}\right)^{n}-|\alpha|\right\} r^{n}\right|\right. \\
& \left.-\left|1-\alpha+\beta\left\{\left(\frac{R+1}{r+1}\right)^{n}-|\alpha|\right\}\right|\right) \mid \\
& \leq\left|P\left(R e^{i \theta}\right)-\alpha P\left(r e^{i \theta}\right)+\beta\left\{\left(\frac{R+1}{r+1}\right)^{n}-|\alpha|\right\} P\left(r e^{i \theta}\right)\right| \\
& +\frac{m}{2}\left(\left|R^{n}-\alpha r^{n}+\beta\left\{\left(\frac{R+1}{r+1}\right)^{n}-|\alpha|\right\} r^{n}\right|\right. \\
& \left.\quad-\left|1-\alpha+\beta\left\{\left(\frac{R+1}{r+1}\right)^{n}-|\alpha|\right\}\right|\right) \\
& \int_{0}^{2 \pi} \mid R^{n}-\alpha r^{n}+\beta r^{n}\left\{\left(\frac{R+1}{r+1}\right)^{n}-|\alpha|\right\} \\
& +e^{i \gamma}\left(1-\bar{\alpha}+\bar{\beta}\left\{\left(\frac{R+1}{r+1}\right)^{n}-|\alpha|\right\}\right) \mid d \gamma \\
& =\int_{0}^{2 \pi} \| e^{i \gamma}\left|1-\bar{\alpha}+\bar{\beta}\left\{\left(\frac{R+1}{r+1}\right)^{n}-|\alpha|\right\} r^{n}+\beta r^{n}\left\{\left(\frac{R+1}{r+1}\right)^{n}-|\alpha|\right\}\right| \\
& \mid R^{n} \\
& \quad \mid \gamma
\end{aligned}
$$

$$
\begin{gathered}
=\int_{0}^{2 \pi} \| R^{n}-\alpha r^{n}+\beta r^{n}\left\{\left(\frac{R+1}{r+1}\right)^{n}-|\alpha|\right\} \mid \\
+\left.e^{i \gamma}\left|1-\alpha+\beta\left\{\left(\frac{R+1}{r+1}\right)^{n}-|\alpha|\right\}\right|\right|^{p} d \gamma \\
=\int_{0}^{2 \pi} \| R^{n}-\alpha r^{n}+\beta r^{n}\left\{\left(\frac{R+1}{r+1}\right)^{n}-|\alpha|\right\} \mid e^{i \gamma} \\
\quad+\left.\left|1-\alpha+\beta\left\{\left(\frac{R+1}{r+1}\right)^{n}-|\alpha|\right\}\right|\right|^{p} d \gamma \\
=\int_{0}^{2 \pi} \mid\left(R^{n}-\alpha r^{n}+\beta r^{n}\left\{\left(\frac{R+1}{r+1}\right)^{n}-|\alpha|\right\}\right) e^{i \gamma} \\
+\left(1-\alpha+\beta\left\{\left(\frac{R+1}{r+1}\right)^{n}-|\alpha|\right\}\right) \mid d \gamma,
\end{gathered}
$$

the desired result follows immediately by using (41) in (40).

This completes the proof of Theorem 1.

Proof of the Theorem 4. Since Theorem 1 holds for every $\beta$ with $|\beta| \leq 1$, taking in particular $\beta=0$, we get

$$
\begin{gathered}
\left\|P(R z)-\alpha P(r z)+\frac{m \delta}{2}\left(\left|R^{n}-\alpha r^{n}\right|-|1-\alpha|\right)\right\|_{p} \\
\leq \frac{\left\|\left(R^{n}-\alpha r^{n}\right) z-(1-\alpha)\right\|_{p}}{\|1+z\|_{p}}\|P(z)\|_{p} .
\end{gathered}
$$

Now by Minkowski's inequality, we have, for every $p \geq 1$,

$$
\begin{aligned}
& \left\|P(R z)+\frac{m \delta}{2}\left(\left|R^{n}-\alpha r^{n}\right|-|1-\alpha|\right)\right\|_{p} \\
& =\| P(R z)-\alpha P(r z) \\
& \quad+\frac{m \delta}{2}\left(\left|R^{n}-\alpha r^{n}\right|-|1-\alpha|\right)+\alpha P(r z) \|_{P} \\
& \leq\left\|P(R z)-\alpha P(r z)+\frac{m \delta}{2}\left(\left|R^{n}-\alpha r^{n}\right|-|1-\alpha|\right)\right\|_{P} \\
& \quad+\|\alpha P(r z)\|_{P} .
\end{aligned}
$$

Using inequalities (28) (of Lemma 11) and (42) in (43), we get, for every $\alpha, \delta \in \mathscr{C}$ with $|\alpha| \leq 1,|\delta| \leq 1, R>r \geq 1$ and $p \geq 1$,

$$
\begin{aligned}
\| P & (R z)+\frac{m \delta}{2}\left(\left|R^{n}-\alpha r^{n}\right|-|1-\alpha|\right) \|_{p} \\
& \leq \frac{\left\|\left(R^{n}-\alpha r^{n}\right) z-(1-\alpha)\right\|_{p}}{\|1+z\|_{p}}\|P(z)\|_{p}+|\alpha| r^{n}\|P(z)\|_{p} \\
& =\left\{|\alpha| r^{n}+\frac{\left\|\left(R^{n}-\alpha r^{n}\right) z-(1-\alpha)\right\|_{p}}{\|1+z\|_{p}}\right\}\|P(z)\|_{p},
\end{aligned}
$$

which is inequality (16) and this completes the proof of Theorem 4. 


\section{Conflict of Interests}

The authors declare that there is no conflict of interests regarding the publication of this paper.

\section{References}

[1] S. Bernstein, "Sur la Limitation Des Derivées Des Polnômes," Comptes Rendus de l'Académie des Sciences, vol. 190, pp. 338341, 1930.

[2] M. Riesz, "Über Einen Sat'z des Herrn Serge Bernstein," Acta Mathematica, vol. 40, no. 1, pp. 337-347, 1916.

[3] P. D. Lax, "Proof of a conjecture of P. Erdös on the derivative of a polynomial," Bulletin of the American Mathematical Society, vol. 50, pp. 509-513, 1944.

[4] N. C. Ankeny and T. J. Rivlin, "On a theorem of S. Bernstein," Pacific Journal of Mathematics, vol. 5, pp. 849-852, 1955.

[5] A. Aziz and Q. M. Dawood, "Inequalities for a polynomial and its derivative," Journal of Approximation Theory, vol. 54, no. 3, pp. 306-313, 1988.

[6] V. K. Jain, "Generalization of certain well known inequalities for polynomials," Glasnik Matematički, vol. 32, no. 1, pp. 45-51, 1997.

[7] A. Aziz and N. A. Rather, "Some compact generalizations of Bernstein-type inequalities for polynomials," Mathematical Inequalities \& Applications, vol. 7, no. 3, pp. 393-403, 2004.

[8] A. Liman, R. N. Mohapatra, and W. M. Shah, "Inequalities for polynomials not vanishing in a disk," Applied Mathematics and Computation, vol. 218, no. 3, pp. 949-955, 2011.

[9] M. Bidkham, H. A. Soleiman Mezerji, and A. Mir, " $L^{p}$ inequalities and admissible operator for polynomials," Analysis in Theory and Applications, vol. 28, no. 2, pp. 156-171, 2012.

[10] A. Aziz and N. A. Rather, " $L^{p}$ inequalities for polynomials," Glasnik Matematički, vol. 52, no. 1, pp. 39-43, 1997.

[11] A. Aziz and N. A. Rather, " $L^{p}$ inequalities for polynomials," Applied Mathematics, vol. 2, no. 3, pp. 321-328, 2011.

[12] A. Aziz and N. A. Rather, "New integral mean estimates for polynomials," Proceedings of the Indian Academy of Science, vol. 109, no. 1, pp. 65-74, 1999.

[13] N. A. Rather, "Some integral inequalities for polynomials," Southeast Asian Bulletin of Mathematics, vol. 33, no. 2, pp. 341348, 2009.

[14] R. P. Boas Jr. and Q. I. Rahman, " $L^{p}$ inequalities for polynomials and entire functions," Archive for Rational Mechanics and Analysis, vol. 11, pp. 34-39, 1962.

[15] A. Aziz and N. A. Rather, "New $L^{p}$ inequalities for polynomials," Mathematical Inequalities \& Applications, vol. 1, no. 2, pp. 177191, 1998.

[16] G. H. Hardy, "The mean value of the modulus of an analytic function," Proceedings of the London Mathematical Society, vol. 14, pp. 269-277, 1915. 


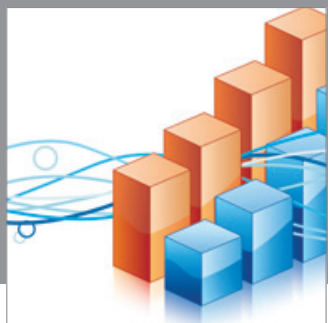

Advances in

Operations Research

mansans

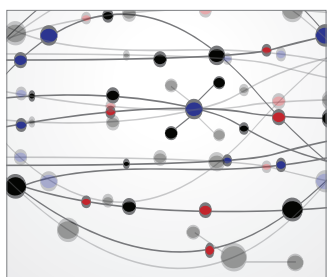

The Scientific World Journal
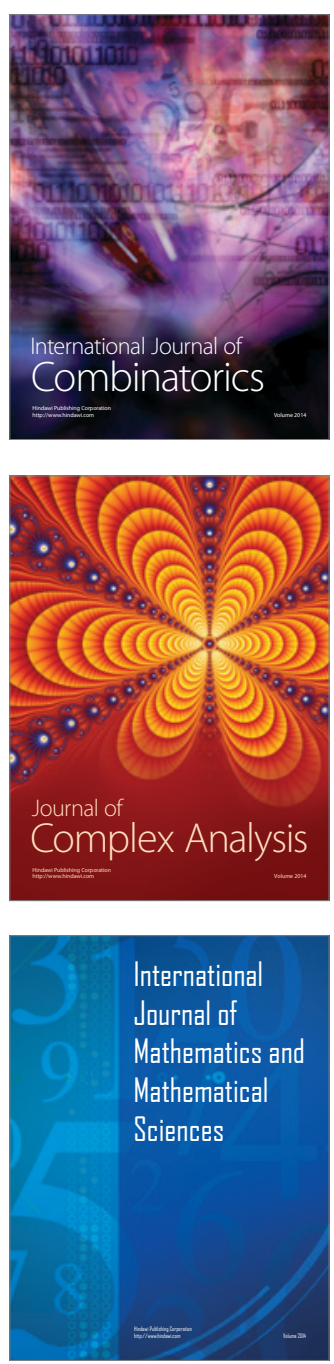
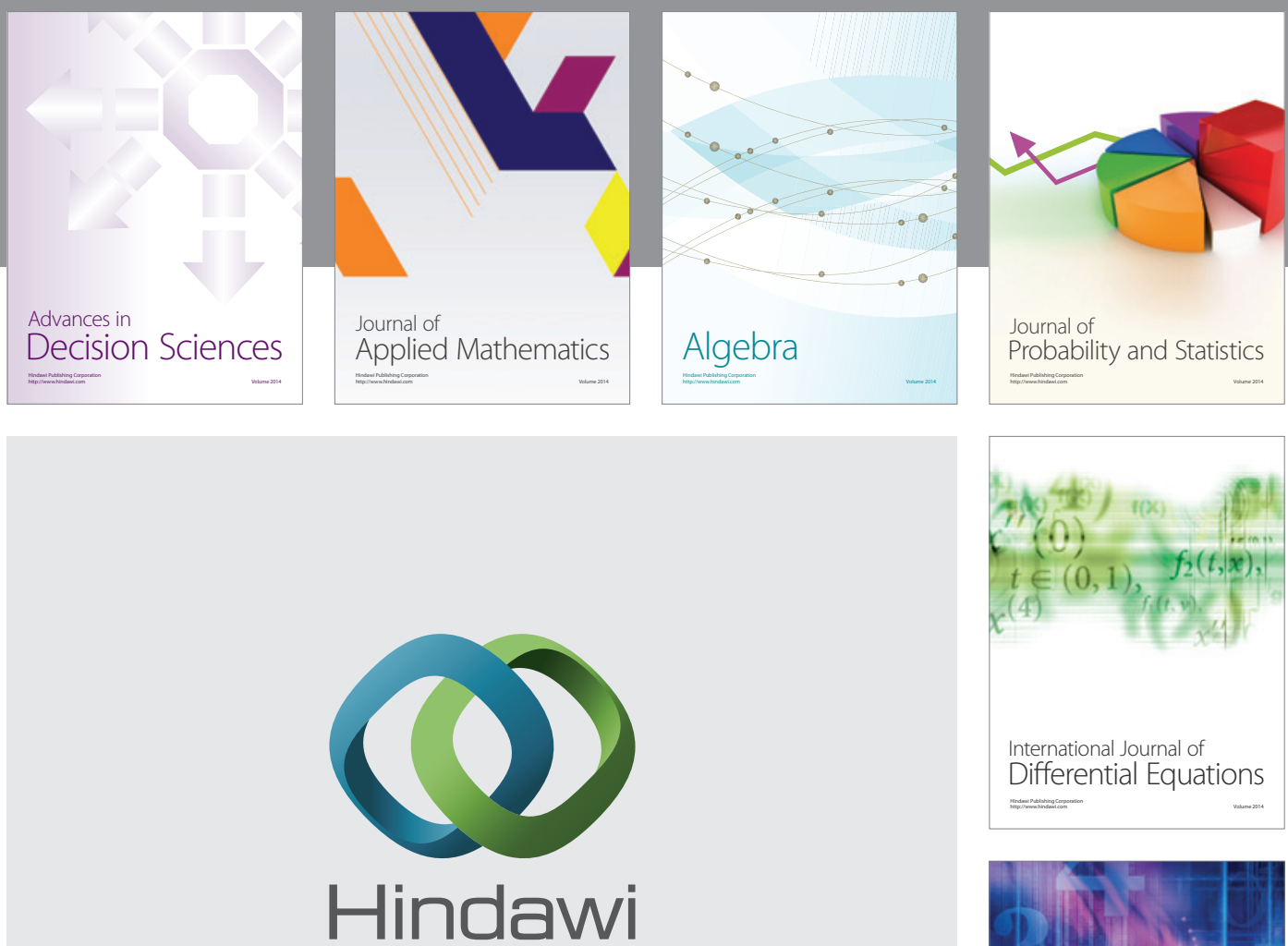

Submit your manuscripts at http://www.hindawi.com
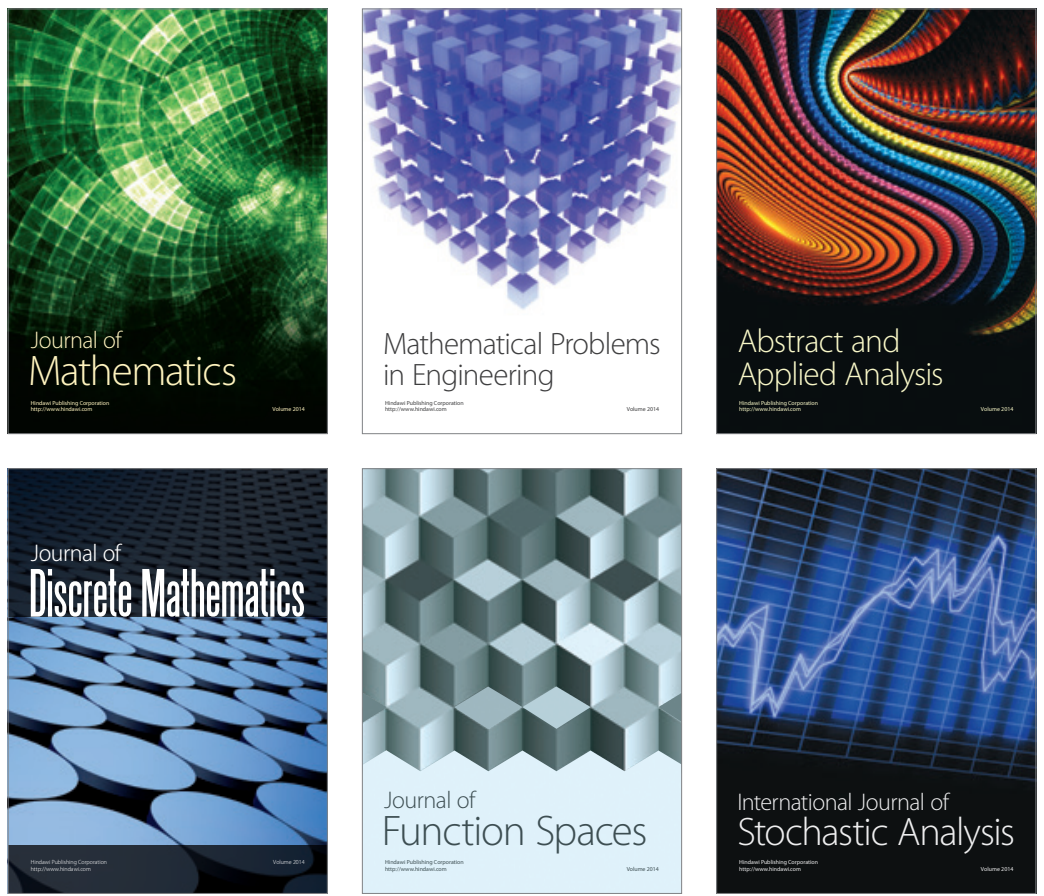

Journal of

Function Spaces

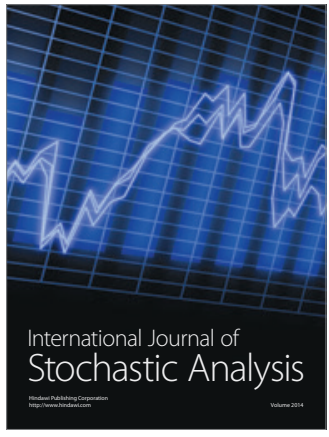

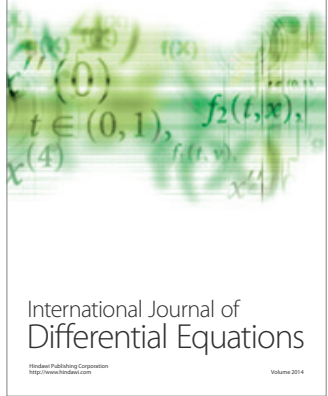
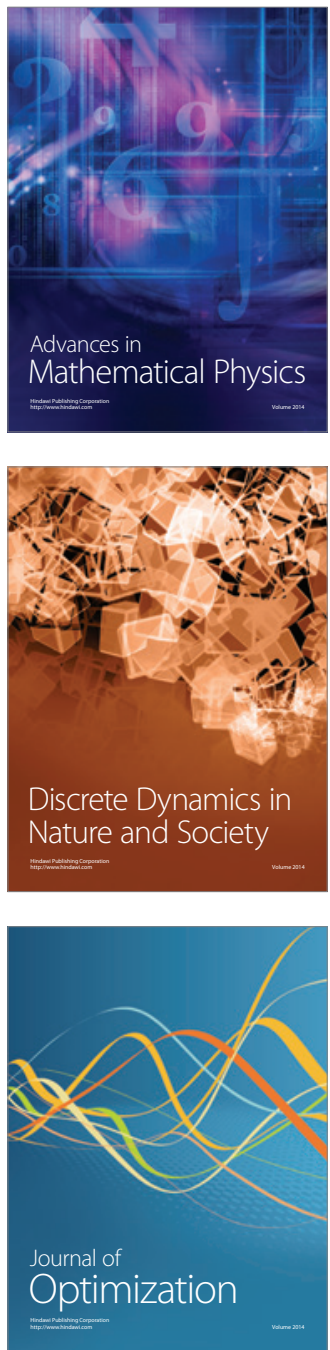